\title{
A SHORT-ORBIT SPECTROMETER FOR HALL C
}

\author{
H. E. Jackson, R.S. Kowalczyk, D. H. Potterveld, B. Zeidman \\ Argonne National Laboratory, Argonne, Illinois, 60439 \\ R. L. Boudrie, V. E. Hart \\ Los Alamos National Laboratory, Los Alamos, New Mexjco, 87545
}

\begin{abstract}
A short-orbit spectrometer capable of detecting particles with momentum $<2.0 \mathrm{GeV} / \mathrm{c}$ with moderate energy and angular resolution will be available in Hall $\mathrm{C}$ for operations in coincidence with the HMS spectrometer or for stand alone operation. The design is based on a $Q D(-D)$ configuration which can meet the needs of a broad spectrum of studies appropriate to Hall C. Measurements will be possible for angles of up to 20 degrees out of plane.
\end{abstract}

\section{Introduction}

The experimental program planned at CEBAF for Hall $\mathrm{C}$ emphasizes coincidence experiments involving a high momentum electron spectrometer and a second arm capable of detecting particles of momenta below $2 \mathrm{GeV} / \mathrm{c}$, with moderate energy and angular resolution. To provide this capability the Argonne National Laboratory Medium Energy Group is building a short-orbit spectrometer, the SOS. The design is based on a $Q D(-D)$ spectrometer which has been developed recently at the Los Alamos Mesor. Physics Facility. The QD(-D) configuration provides a large momentum acceptance, with good energy resolution and solid-angle acceptance in a very compact geometry. The design can be easily adapted to out-of-plane operation. In order to allow measurements at small angles and also, in first order, to decouple the momentum determination from the measurement of scattering angle, the spectrometer will bend in the vertical direction. The basic design is point-to-point focussing in both the radial. and transverse directions. The dipole edges are rotated to provide both radial and transverse focussing and the curvatures of the edges provide higher order aberration corrections.

The SOS, shown schematically in Fig. 1, corsists of thrie basic elements: a quadrupole that focuses in the transverse(horizontal) direction; a dipole that bends the central trajectory $33^{\circ}$; and a second dipole that bends the central trajectory $15^{\circ}$ in the opposite direction. The quadrupole focuses in the transverse direction and is the primary element that maintains acceptance in the horizontal plane. The rotation of the edge angles of the first dipole provides radial focussing (traniverse defocussing) at both the entrance and the exit of the dipole while the curvature of the entrance edge introduces sextupole corrections that act primarily upon the geometric variables. The second dipole, bending in

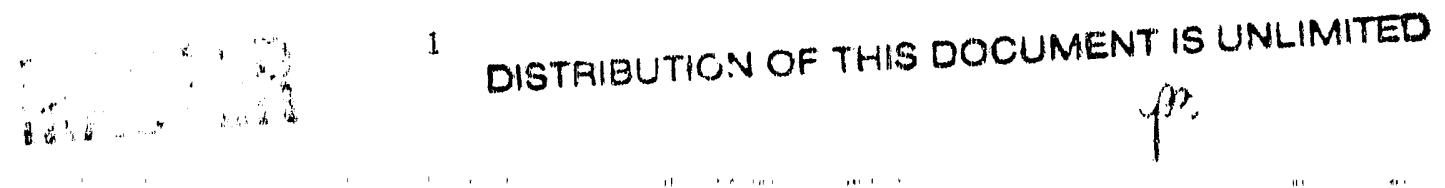


the reverse direction, is quite short and has rotated and curved edges at both the entrance and exit. This dipole, roughly triangular in shape, acts primarily as a quadrupole doublet; the entrance edge focuses radially while the exit edge focuses transversely. Several features of this design are unique. The large rotations of the pole edges provide strong focussing over the full width of the beam, while the use of a reverse bend allows the separation between the two dipoles to be quite small while still retaining the radial quadrupole action at both of the interior edges. This strong focussing results in a short distance from the exit of the second dipole to the nominal focal point and, overall, a very short optical length, $\sim 7.4 \mathrm{~m}$, for the momentum range to be covered. The optical design is quite flexible and may be retuned for special purposes. For example, a point to parallel tune provides increased acceptance in target width at reduced spectrometer soi:d angle.

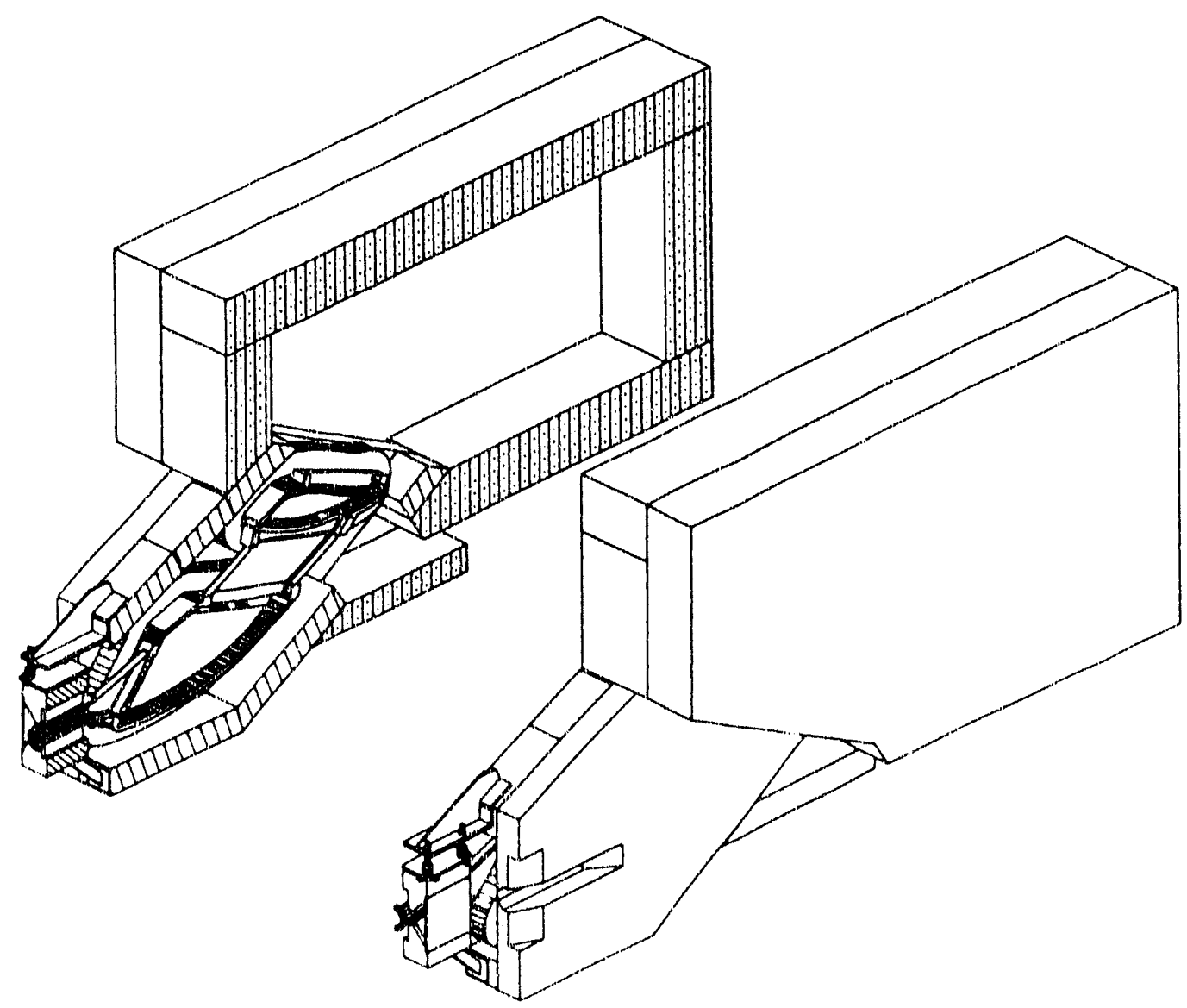

Figure 1.

The SOS Spectrometer and Detector Hut 


\section{Spectrometer Carriage and Shielding}

The SOS spectrometer and shield house carriage will be a motor-powered wheel assernbly with three point suspension. At the focal point, the carriage is supported by a cross-roller bearing assembly on the hall $C$ center pivot as shown in Fig. 2. The rear of the carriage will be supported during positioning by two wheel assemblies which ride on a circular track with a bend radius of 35 feet centered on the Hall $\mathrm{C}$ pivot point. Electromechanical screw jacks will be used to level and position the spectrometer. In order to maximize small angle capability, the forward corners of the dipole yoke are notched to accommodate a beam pipe, thereby permitting the SOS to operate at angles as small as $11.4^{\circ}$ with respect to the incidert electron beam. The maximum scattering angle for SOS will be limited to $164.4^{\circ}$ by Hall $\mathrm{C}$ utility lines.

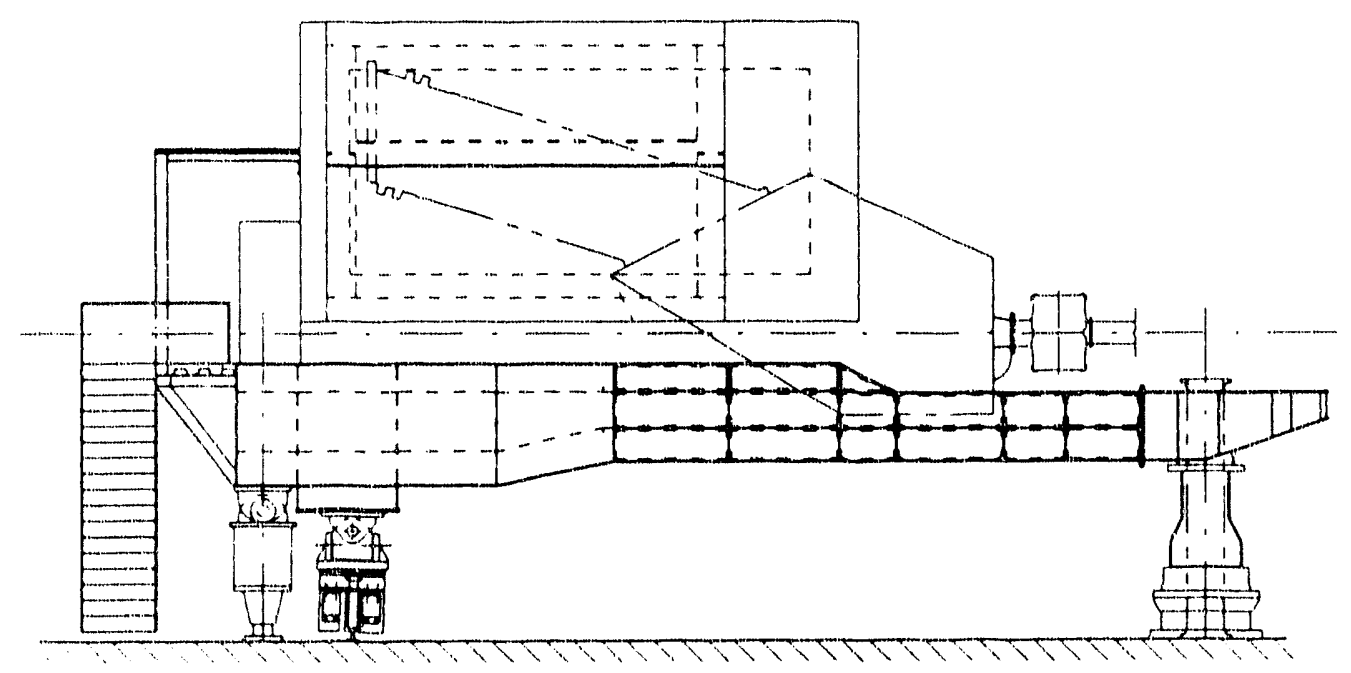

Figure 2.

The SOS Carriage and Shielding Assembly

The SOS carriage structure is designed to ride above the carriage of the HMS spectrometer, thereby allowing a "scissored" interleaving of the two carriages. In addition, the SOS dipole yoke will be chamfered at the front edge to minimize overlap with the cryostat of the first quadrupole of HMS. With these features, the minimum opening angle between SOS and HMS will be $\sim 23^{\circ}$. The SOS carriage will ride on a spherical bearing with a radius of $1 \mathrm{~m}$ centered on the spectrometer focal point. Rotation of the complete SOS assembly out of the horizontal plane by angles of up to $20^{\circ}$ will be possible. Coarse out-of-plane(OOP) adjusiments will be made by a hydraulic jack system located at the rear of the carriage with 
final trimming of the vertical positioning being accomplished, with an electromechanical jack. Limitations on the range of OOP operation will be imposed by the SOS-beam line and the HMS-SOS interfaces. OOP operation will be possible for HMS-SOS opening angles $>60^{\circ}$, with urrestricted OOP operation possible for opening angles $>66^{\circ}$. Beam line interference will limit OOP unrestricted operation of SOS to scattering angles $>35^{\circ}$ with limited operation for angles $>\sim 16^{\circ}$.

The shielding structure for the SOS detector system will provide a cavity at the exit of SOS with approximate dimensions, $3.7 \mathrm{~m}$ along the central orbit, $>1.6 \mathrm{~m}$ perpendicular to the bend plane centered on the central orbit, and a vertical space of $\sim 3 \mathrm{~m}$ containing the central orbit. The cavity volume will be lined along its exterior with $5 \mathrm{~cm}$ of lead. Exterior to the lead walls will be additional shielding in the form of boron loaded concrete whose thickness will be $60 \mathrm{~cm}$ except where obstructed by the floor. The structure will include on the side away from the beam a vertical-sliding double door which can be rapidly opened to provide almost total access to the detector assembly from the side. Detector components whose positioning is critical will be rigidly attached to the dipole yoke. The angular pointing tolerance of the optical axis of the spectrometer with respect to the pivot point will be $0.3 \mathrm{mr}$ vertically and in azimuth, while the tolerance in azimuthal placement will be $2.0 \mathrm{mr}$.

\section{SOS Performance}

The momentum resolution of SOS will be limited by measurement errors and aberrations. A reasonable estimate of wire-chamber and, hence, position resolution at the focal plane of SOS is $\sim 0.1 \mathrm{~mm}$. An estimate which includes major aberrations and measurement errors gives a realistic momentum resolution of $<8 \times 10^{-4}$ for a large fraction of the acceptance of the SOS. If there are significant advances in detector technology, it might be possible to improve the momentum resolution. Among the proposals that will use SOS are several that require extended targets several centimeters long, such as $L D$. Targets as long as four cm transverse to the central ray can be viewed with nearly the full solid angle( $9 \mathrm{msr})$ and longer targets can be accommodated for experiments that can accept a reduced solid angle depending on the tune. The basic parameters for SOS are summarized in Table 1. The solid angle given there is for a point source. Under typical experimental conditions, the SOS should operate at luminosities in excess of $10^{38} \mathrm{~cm}^{2} \mathrm{sec}$. Possible luminosity limits generated by backgrounds in the detector package that arise from bearn-generated electromagnetic processes in the primary target were explored in Monte Carlo simulations. The results establish that the event rate due to target associated background is below the rates which will arise from genuine inclusive electron/positron, proton, and pion cross sections. Consequently, it is reasonable to assume that the luminosity limit for the SOS will be imposed by real inclusive particle spectra rather than 
incoherent background radiation. Inclusive cross sections for a carbon target indicate that the luminosity limit will be in excess of $10^{38} / \mathrm{cm}^{2}$ : ec.

Table 1. Short-Orbit Spectrometer Parameters

\begin{tabular}{|c|c|c|c|}
\hline \multirow{14}{*}{ SPECTROMETER: } & Configuration & & $\mathrm{QD}(-\mathrm{D})$ \\
\hline & Horizontal Open. Angle & & $120 \mathrm{mr}$ \\
\hline & Vertical Open. Angle & & $80 \mathrm{mr}$ \\
\hline & Solid Angle & & $9 \mathrm{msr}$ \\
\hline & Focal Plane Dimensions & & $40 \times 120 \mathrm{~cm}$ \\
\hline & Tilt Angle & & $70 \mathrm{deg}$ \\
\hline & Central Momentum at $B_{\text {nom }}$ & & $1.5 \mathrm{GeV}$ \\
\hline & Momentum Acceptance & & $40 \%$ \\
\hline & Dispersion & & $0.92 \mathrm{~cm} / \%$ \\
\hline & Optical Length & & $7.4 \mathrm{~m}$ \\
\hline & $\mathrm{D} / \mathrm{M}$ & & $2.2 \mathrm{in}$ \\
\hline & Angular Range & & $11-165 \mathrm{deg}$ \\
\hline & Total Weight & & 425 tons \\
\hline & Distance, target-Q1 & & $1.5 \mathrm{~m}$ \\
\hline \multirow[t]{3}{*}{ QUADRUPOLE: } & Magnetic Length & & $0.8 \mathrm{~m}$ \\
\hline & Aperture & & $25 \mathrm{~cm}$ \\
\hline & $\mathrm{B}_{\text {nom }}$, Pole Tip & & $1.0 \mathrm{~T}$ \\
\hline \multirow[t]{6}{*}{$\operatorname{DIPOLE}(\mathrm{S}):$} & Gap & & $16 \mathrm{~cm}$ \\
\hline & Pole Face Rot., Entr. & .40 .6 & $-40.6 \mathrm{deg}$ \\
\hline & Exit & -40.6 & $+45.9 \mathrm{deg}$ \\
\hline & Radius Curvature Exit & -2.5 & $2.25 \mathrm{~m}$ \\
\hline & Bending Radii & 2.94 & $2.94 \mathrm{~m}$ \\
\hline & Bend Angles & 33 & $-15 \mathrm{deg}$ \\
\hline
\end{tabular}

This work is supported by the U. S. Department of Energy, Nuclear Physics Division, under contract W-31-109-ENG-38.

DISCLAIMER

\footnotetext{
This report was prepared as an account of work sponsored by an agency of the United States Government. Neither the United States (jovernment nor ary agency thereof, nor any of their employees, makes any warranty, express or implied, or assumes any legal liability or responsibility for the accuracy, completeness, or usefulness of any information, apparatus, product, of process disclosed, or represents that its use would not infringe privately owned rights. Reference herein to any specific commercial product, process, or service by trade name, trademark, manufacturer. or otherwise does not necsessarily constitute or imply its endorsement, recom. mendation, of favoring by the unted states uovernment or any agency thereot the views and opinions of authors expressed herein do not necessarily state or reflect those of the United States Government or any agency thereof.
} 

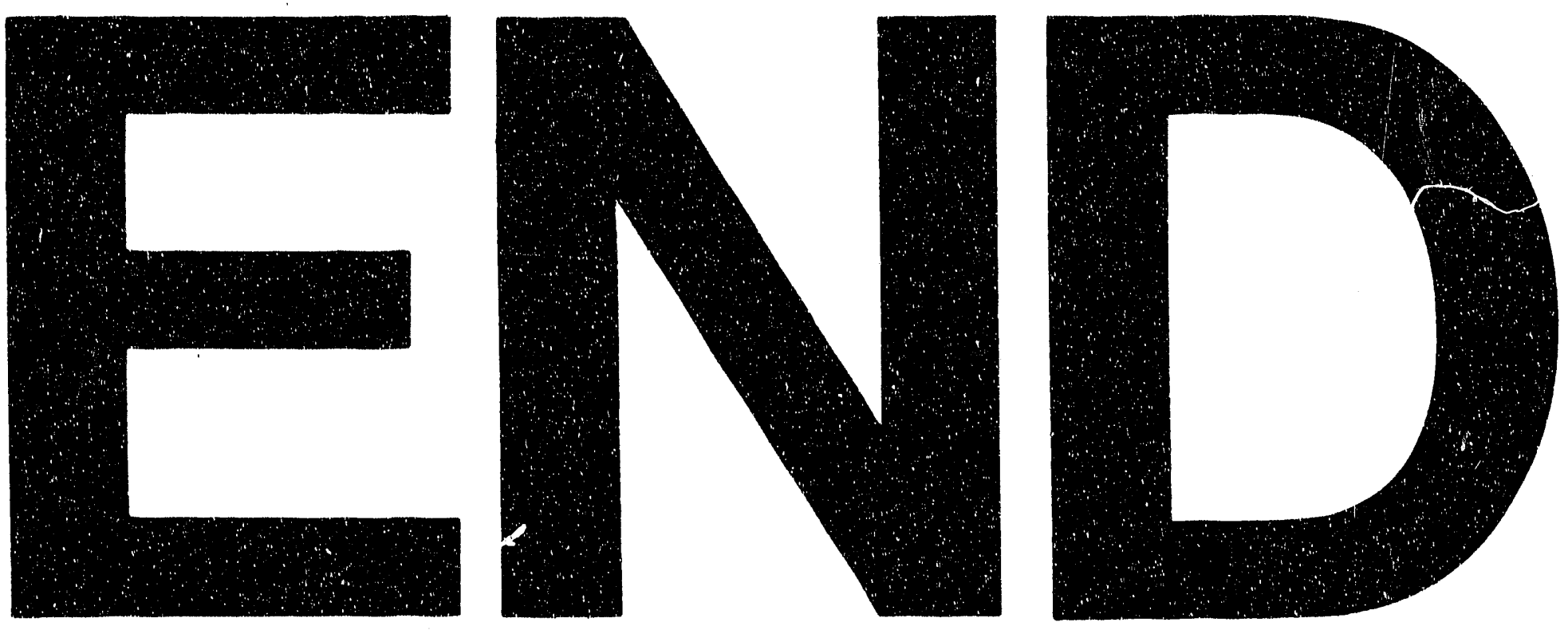

$\$$
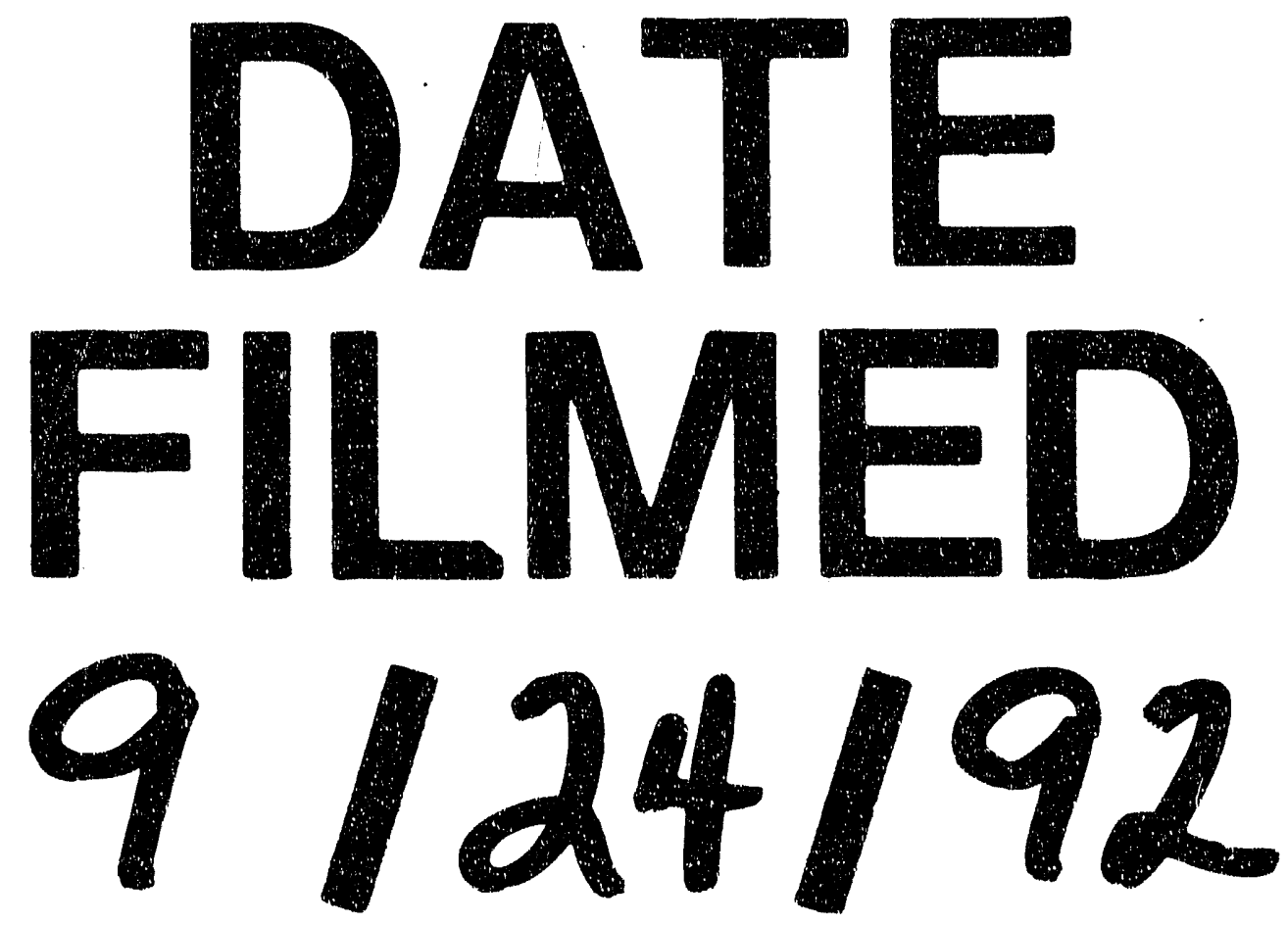
